\title{
Modeling of hydraulic axial piston pumps including specific signs of wear and tear
}

\author{
Christian Bayer Olaf Enge-Rosenblatt \\ Fraunhofer Institute for Integrated Circuits, Division Design Automation \\ Zeunerstrasse 38, 01069 Dresden, Germany \\ \{Christian.Bayer; Olaf.Enge\}@ eas.iis.fraunhofer.de
}

\begin{abstract}
Reliability of machines and facilities has played an important role since many years. Nowadays, attention is also paid to maintenance times. Maintenance standstills are to be reduced as far as possible. On the other hand, technical systems are subject to signs of wear and tear which are in general growing slowly and imperceptibly. The gradual abrasion of applied tools may lead to poor production tolerances or to a component's standstill. Hence, a condition-based maintenance strategy will be of increasing importance. Such a strategy requires a permanent condition monitoring during operation. To this end, reliable high-performance algorithms for signal processing, feature extraction, and classification are needed. Modeling the process of wear and tear may be useful to find the particular steps of the condition monitoring system's signal processing. This strategy was investigated by means of one very important device from automation engineering, a hydraulic axial piston pump. The procedure of getting signals by an appropriate Modelica model of the main parts of the pump is shown within the paper. Additionally, the manipulation process for the signals and the steps of classification are shortly presented to give an overview to the possibilities of model-based signal generation based on a Modelica model. The advantages of the multi-physics modeling language are emphasized because the axial piston pump model combines the mechanical and the hydraulic domain in a very efficient way.
\end{abstract}

Keywords: condition monitoring; classification; signal processing

\section{Introduction}

Long-lasting correct operation and the highest level of availability are important requirements concerning machines and facilities in today's industry. While reliability has played an important role since many years, more and more attention has been paid to the times needed for maintenance processes in the last decade. The number of maintenance standstills has to be reduced to decrease the total cost of ownership (TCO). This is enforced by the increasing business rivalry of today's economy. On the other hand, technical systems are subject to a proceeding deterioration and often to a certain wear. But signs of wear and tear are in general growing slowly and imperceptibly. The gradual abrasion of applied tools may lead little by little to poor production tolerances or even to a standstill of a component or a complete production facility. Hence, a condition-based maintenance strategy will be of increasing importance. Such a strategy requires a permanent condition monitoring during operation based on efficient behavior analysis procedures. Condition monitoring systems analyze various measured signals using application-specific algorithms. In this context, reliable highperformance algorithms for signal processing, feature extraction, and classification are needed.

Modeling the process of wear and tear may be useful to find the particular steps of signal processing which are suitable for a certain condition monitoring system. This strategy was investigated by means of a hydraulic axial piston pump, which is a very important device from automation engineering and is still an intense subject of computer aided modeling $[1,2]$.

In section 2, two models of the standard pump are explained first. Both models combine a mechanical and a hydraulic sub-model. Physically motivated inter-modular connections are established. In section 3 , a parameter optimization method is presented and discussed. This method is applied to fit unknown parameters of the mechanical sub-model. Measured time signals are used for comparison. Afterwards, some signs of wear are implemented in the model. This is presented in section 4. Two of the most problematic cases leading to the main failures are shown. Characteristic features deduced from Fourier transforms of vibration signals are used for classification of good pumps and pumps having failures. 


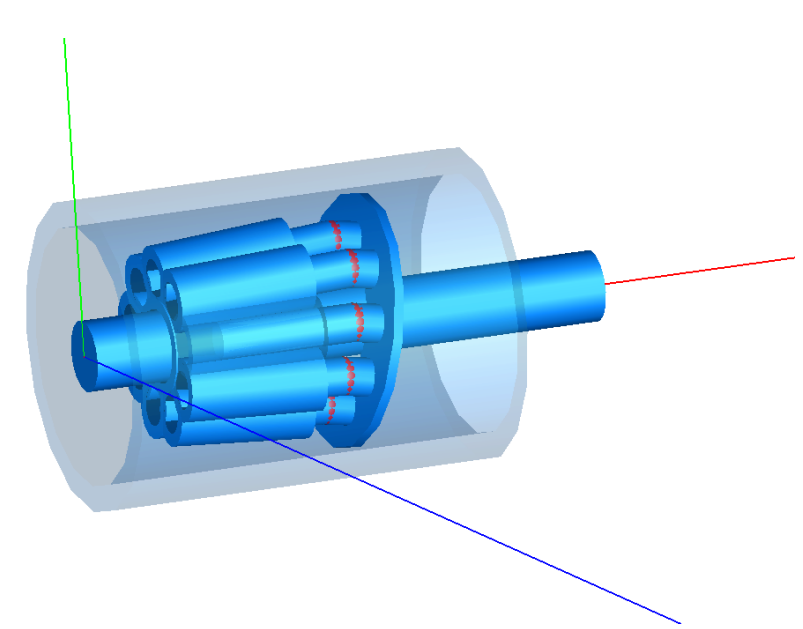

Figure 1: Schematic of the modeled axial piston pump with 9 cylinders. Only mechanical parts are shown.

\section{Modelica model}

\subsection{The axial piston pump}

For demonstration of the above mentioned condition monitoring approach we chose an axial piston pump. Those devices basically consist of a certain number of cylinders and pistons to pump hydraulic oil under high pressure conditions. The cylinders are evenly aligned around a rotating axis, whereas the pistons are attached to a tilted cam, which is called swash plate. Due to rotation, the pistons can perform an axial motion and thus pump the oil through inlet and outlet ports respectively. Figure 1 shows a rough schematic of the pump, which is captured from a 3D Dymola animation [3]. The oil flow is controlled by a distributor plate, which e.g. connects the outlet port to the cylinders with high oil pressure. The plate is not shown in Figure 1 as the flow control is implemented within the hydraulics model.

\section{$2.23 D$ model}

Modelica is capable of simulating multi-body dynamics as well as models based on user-specific differential equations. A good reference of the Modelica language can be found in [4].

Our initial model was a spatial model (3D) exploiting the MultiBody package for mechanics and using further differential equations for hydrodynamics. Unfortunately, a lot of parameters, for example certain friction coefficients or spring rates, are not known exactly, even not by the manufacturer. Hence, the model must be suited for parameter optimization algorithms in terms of computational effort. Since the $3 \mathrm{D}$ model is very complex, we reduced the model

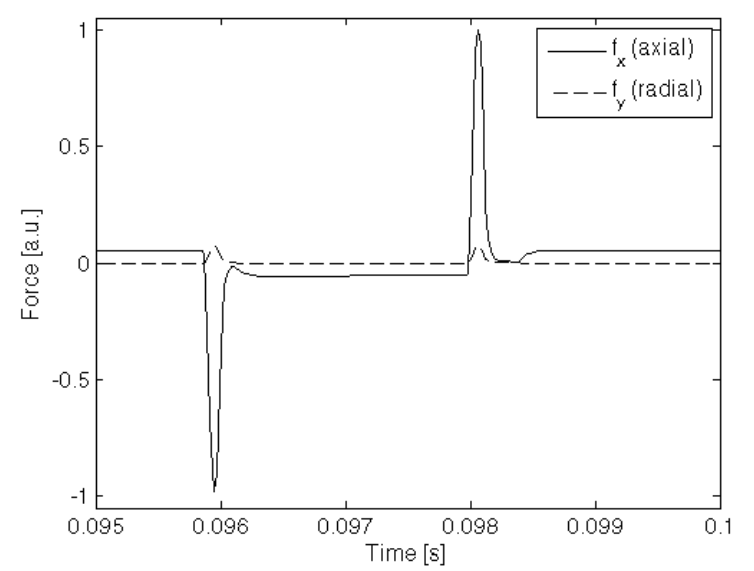

Figure 2: Comparison of axial and radial forces acting on the housing. The results are based on the 3D model.

to the very essential parts and developed a 1D equivalent. To justify this approach we evaluated the forces acting to the pump's housing. The device rotated at constant speed of 1500 revolutions per minute. Figure 2 shows axial and radial forces during a section of $1 / 9$ of a full revolution of the pump. Obviously, the translational motion of the pistons is the main source of vibration, which is the value we want to measure and simulate. In an ideal case, the rotation itself does not contribute to vibration. Only the slight tilt of the cylinders against the rotational axis causes a radial force, which is directly related to the sine of the tilt angle. Since this angle is small, the radial force can be neglected. Therefore, the reduced model considers axial motion only and thus becomes a 1D representation.

Since we focused on the 1D model, the 3D implementation will not be explained in detail here.

\subsection{D model}

The reduced mechanical model is depicted in Figure 3. Each element of the schematic relates to a dedicated Modelica sub-model, which in turn is described by certain differential equations and an individual set of parameters. Our 1D model complies with the conventions of Modelica's translational mechanics library and we also used a few basic components thereof. The elements are chained exactly as shown in Figure 3 and form lines or parallel lines respectively.

The housing of the pump is modeled as a simple mass, which embraces all other parts of the model. It is therefore connected in parallel to both sides of the pump's interior element chain. The mass is not fixed and its movement gives rise to the acceleration value and thus vibration. On the left side of the chain a 


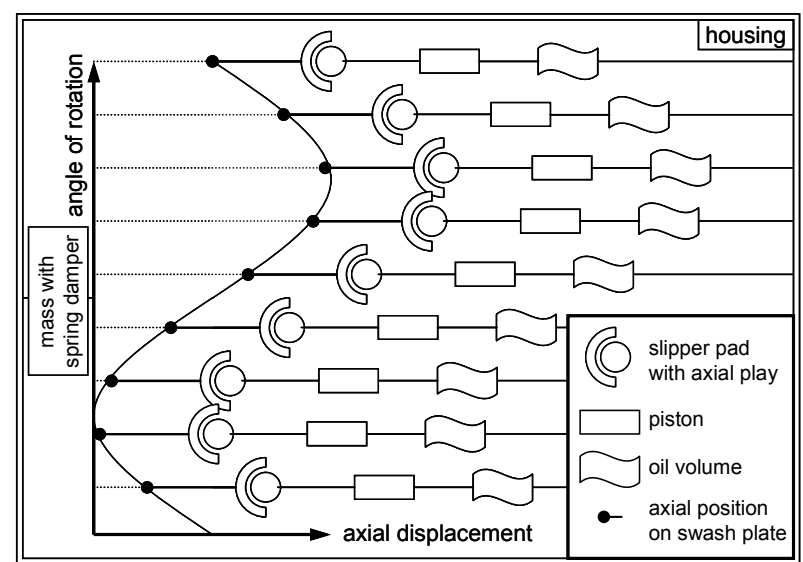

Figure 3: Reduced 1D model of the axial piston pump.

mass with spring damper follows the housing. It sums up certain masses inside the pump and connects them to the housing by means of a spring. This indeed approximates the real assembly of the pump.

The next element in the row is the tilted swash plate, represented by the sine curve in Figure 3. It causes all pistons to follow a sinusoidal motion with individual phase offset. This effect is implemented by time dependent displacements as follows:

$$
\begin{aligned}
0 & =F_{a}+F_{b} \\
s_{\text {rel }} & =s_{b}-s_{a} \\
& =\hat{s}(1-\cos (\varphi)) \\
\varphi & =2 \pi \frac{N}{60} t+\varphi_{\text {piston }}
\end{aligned}
$$

$F$ denotes forces at the sub-models flanges and $s$ is the distance between flanges or the position of them. $\varphi$ is the absolute revolution angle of the pump. The swash plate model can only drive one of the 9 pistons with specific phase offset. At this point the model chain breaks up into 9 individual lines in parallel. Each line consists of a swash plate model followed by slipper pad, piston and the cylinder oil volume. All 9 lines finally merge into the right hand frame of the housing mass.

The connection between piston and swash plate is established by slipper pads. They offer a tight connection to the piston with small play, which extends as a result of wear. The sub-model allows a small distance of free motion until the piston hits one of the two limiting boundaries. Once in contact with the limitation, the model switches to a damped spring mass system behavior with spring rate $D$, as can be seen in the equations below.

$$
\begin{aligned}
F & =-F_{a}=F_{b} \\
s_{\text {rel }} & =s_{b}-s_{a} \\
F & =\left\{\begin{array}{lll}
s_{\text {rel }}<0 & : & R_{1} \frac{\partial}{\partial t} s_{\text {rel }}+D_{1} s_{\text {rel }} \\
s_{\text {rel }}>s_{\text {gap }} & : & R_{2} \frac{\partial}{\partial t} s_{\text {rel }}+D_{2}\left(s_{\text {rel }}-s_{\text {gap }}\right) \\
\text { otherwise } & : & 0
\end{array}\right. \text { lll } \\
R_{1} & =\left\{\begin{array}{lll}
v>0 & : & 0 \\
\text { otherwise } & : & R_{1}^{*}
\end{array}\right. \\
R_{2} & =\left\{\begin{array}{lll}
v<0 & : & 0 \\
\text { otherwise } & : & R_{2}^{*}
\end{array}\right.
\end{aligned}
$$

$R$ corresponds to friction coefficients and $v$ to the velocity of the related piston. It turned out, that the simulation results improve, if there is no friction when the piston pulls apart from a boundary. The equations for $R_{i}$ therefore depend on the sign of $v$.

The piston itself is a simple mass of length $L$ with friction and inertia. Stribeck friction is not used in the model as it would introduce too many unknown parameters. The piston equation reads

$$
\begin{aligned}
F_{a}+F_{b} & =m \frac{\partial^{2}}{\partial t^{2}} s_{a}+R \frac{\partial}{\partial t} s_{a} \\
s_{r e l} & =s_{b}-s_{a}=L
\end{aligned} .
$$

The sub-model of oil volume $V$ approximates the conditions within a cylinder and finally links hydraulic quantities to the mechanical part of the model. In [5] this was done by incorporating two different software packages for hydraulics and mechanics. We used Modelica for both physical domains to merge them into one model.

Pressure $p$ inside a cylinder causes fluid flow $q$ through the ports as well as force to a piston's flange. The oil is considered as compressible fluid with bulk modulus $K$. It flows either through the inlet or the outlet port, which depends on the relative angular position of cylinder block and distributor plate. Both ports are modeled as valves with variable cross sections and, thus, have time dependent hydraulic conductance $G$. For a single cylinder the valves' cross sections are shown in Figure 4. Other cylinders experience the same characteristic, but with a phase offset. The model assumes turbulent flow through a valve [6], which finally leads to the equations 


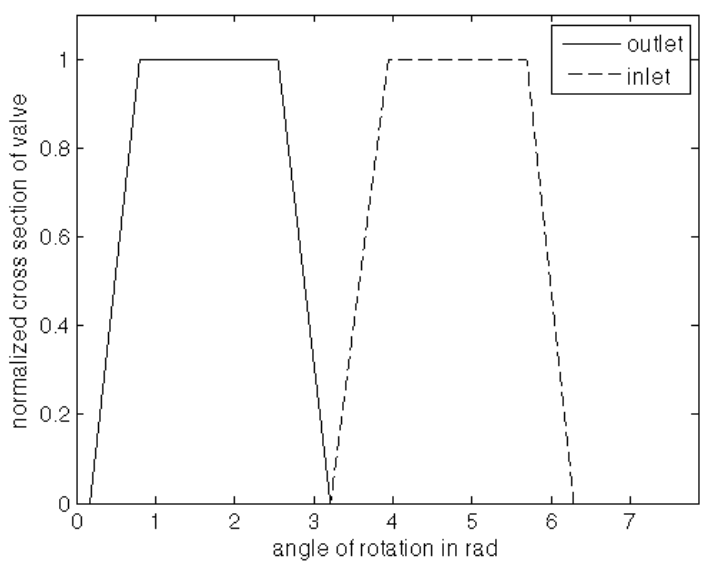

Figure 4: Representative cross sections of inlet and outlet valves for a single cylinder. The graph shows conditions for one revolution of the pump.

$$
\begin{aligned}
s_{\text {rel }} & =s_{b}-s_{a} \\
V & =S_{\text {rel }} A_{\text {piston }} \\
q & =G_{\text {out }} \operatorname{sgn}\left(P_{\text {max }}-p\right) \sqrt{\left|P_{\text {max }}-p\right|}+\ldots \\
& +G_{\text {in }} \operatorname{sgn}\left(P_{\text {min }}-p\right) \sqrt{\left|P_{\text {min }}-p\right|} \\
Q & =q+A_{\text {piston }} \frac{\partial}{\partial t} S_{\text {rel }} \\
\frac{\partial}{\partial t} p & =K \frac{Q}{V} \\
F & =F_{a}=-F_{b}=p A_{\text {piston }}
\end{aligned}
$$

The time dependent cross sections of inlet and outlet are provided by the manufacturer. They were implemented by using Modelica's look-up table component. As can be seen from equation (4), the oil volume sub-model has two frames with variables $s$ and $F$. Hence, it can be easily connected to our chain of other mechanical translational components.

\section{Parameter optimization}

\subsection{Method}

Each sub-model has an individual set of parameters. Some of them are known, as is typically true for geometric parameters. Others have to be estimated or can be found by optimization. For this purpose, several measurements with at least one undamaged pump have to be done. With this reference data, an optimization algorithm was used to find an appropriate parameter set. This is a common method and was also applied in [7] for example. We used MATLAB ${ }^{\circledR}[8]$ for an automated parameter sweep in Dymola. The goal function for minimization is sim-

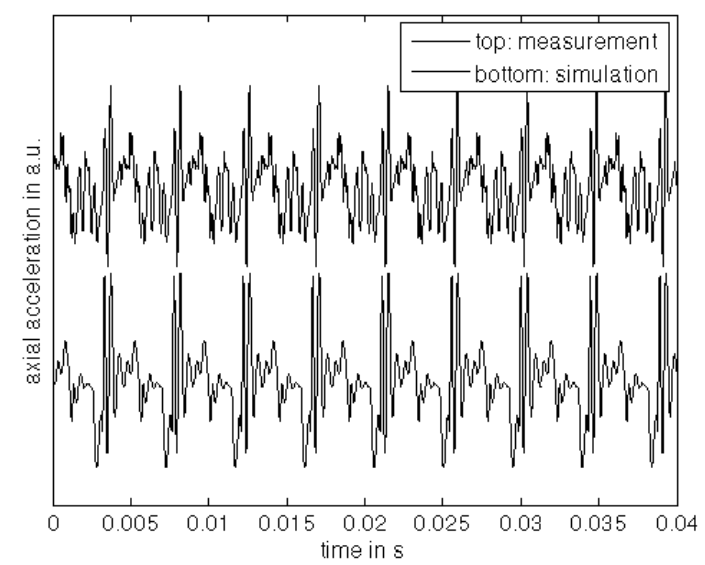

Figure 5: Comparison of simulation results with optimized parameter set and averaged measurement. The signals correspond to the time resolved acceleration of the housing in axial direction. The graph shows one revolution of the pump.

ply the deviation of the simulation result from the averaged measurement signal. The measurand equals in both cases the acceleration of the housing in axial direction. Unfortunately, the number of parameters is quite high, which impedes a reasonable optimization process. Within our reduced 1D model we identified seven parameters, which significantly influence the result and were used for optimization: The simulation is very sensitive to the interaction of piston head and slipper pad. Already four parameters can be extracted from this knowledge, two for each limiting boundary. When hitting a boundary, the piston will penetrate into the material of the slipper pad, squeeze the oil film between pad and swash plate or strain the pad. An easy but efficient way is to assume a springlike model with damping for that process. This gives two parameters for each boundary, namely spring rate and friction coefficient. Another important parameter is the friction of the piston within the cylinder. The last two parameters belong to the springmass system, which is located between housing and swash plate (see Figure 3).

One problem of the optimization is that there is no unique ideal parameter set. The results differ with the initial parameters. To account for this, we also implemented a random sweep of starting values. Once a good parameter set is found, the actual optimization is performed. The algorithm used here was the simplex search method.

\subsection{Results}

In spite of the complicated optimization process, we found a set which fits the real measurement quite well. Figure 5 shows the result. The signal itself has 


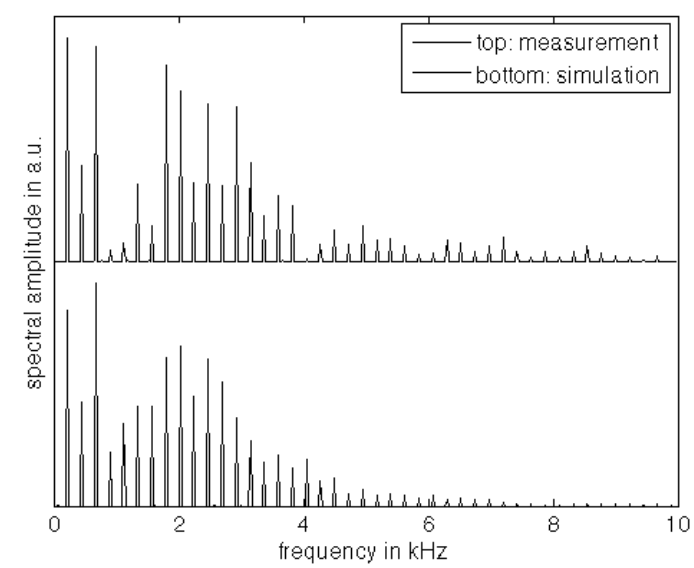

Figure 6: Spectra of simulation result with optimized parameter set and averaged measurement. The spectra correspond to the Fourier transform of the acceleration of the housing in axial direction.

some clear characteristics. During one revolution of the pump, each piston accomplishes one period of its sinusoidal movement. This leads to 9 equally shaped pulses in the time signal. Both, simulated and measured signal, show this characteristic and are comparable.

The axial piston pump is a rotating device. For condition monitoring of this special application the spectrum and evaluation of harmonics is far more interesting than the time signal. Hence, the consistency of simulated and measured signal in the frequency domain is very important. Figure 6 compares the results. Due to the 9 pistons and the rotational frequency of $25 \mathrm{~Hz}$, harmonics with a spacing of $225 \mathrm{~Hz}$ can be observed. Both spectra show a similar distribution. However, within the measurement data harmonics 4 to 7 seem to be rather suppressed, which holds for all measurements, even for different pumps with varying signs of wear. This effect might be caused by the experimental setup. The pump is attached to a motor and other machinery which can vibrate as well. Our model does not consider the exact environmental conditions and is not able to reproduce the harmonics suppression. But it turned out, that we can simply ignore these harmonics for classification.

\section{Modeling defects}

\subsection{Introducing defects}

The main purpose of the simulation is the estimation of measurement signals for worn pumps. Once the model parameters are optimized for an undamaged device, they can be modified slightly to introduce

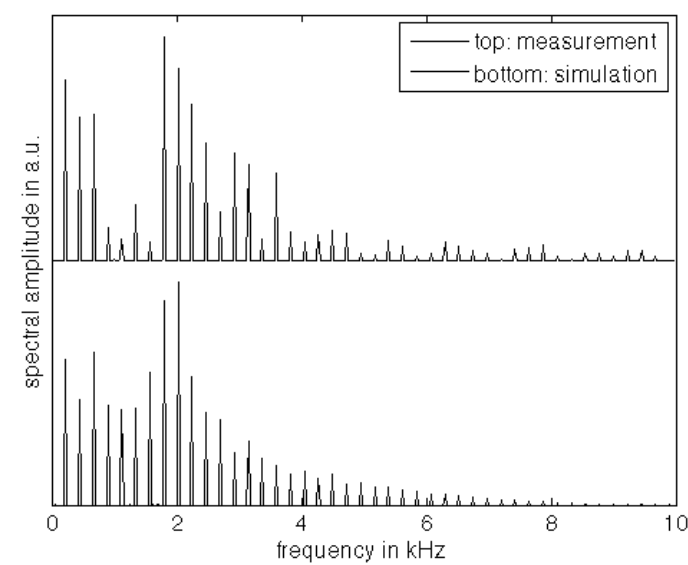

Figure 7: Spectra of measurement signals of a worn pump with axial piston play and simulation result.

defects. As a matter of course this requires the parameters to be related to certain signs of wear.

We investigated two types of defects. One of them is cavitation pitting, which influences the hydraulic conductance of the cylinder's valves. Basically, the cross section of each valve changes slightly with gradual abrasion. Worn pumps give suitable information on the modified cross section and we simply exchanged the characteristic area functions, shown in Figure 4. However, our results suggest that a reasonable change of the functions has only a minor effect to the axial vibration within the considered frequency range. This is consistent with measured data, where the difference is present but comparably small. In the following we focus on a more significant defect.

Each piston is attached to a slipper pad with a certain amount of play. This small gap increases with time and leads to a defect referred to as axial piston play (app). We introduced this effect in the slipper pad sub-model by increasing the play parameter and decreasing one of the spring rates, which models the strain of the pad when pulled by the piston. Figure 7 compares measurements of a worn pump with axial piston play and our simulation results. Since the classification is done in the spectral domain, only the Fourier transforms are shown. Ignoring harmonics 4 to 7 , we can see a very similar trend of change in both the simulation and measurement. The first 3 harmonics drop in amplitude, while others near $2 \mathrm{kHz}$ increase. Based on such effects unique features for classification can be found.

\subsection{Classification}

The classification method used here is based on the first 17 harmonics of the signal's spectrum excluding harmonics 4 to 7. Several statistical parameters, like 


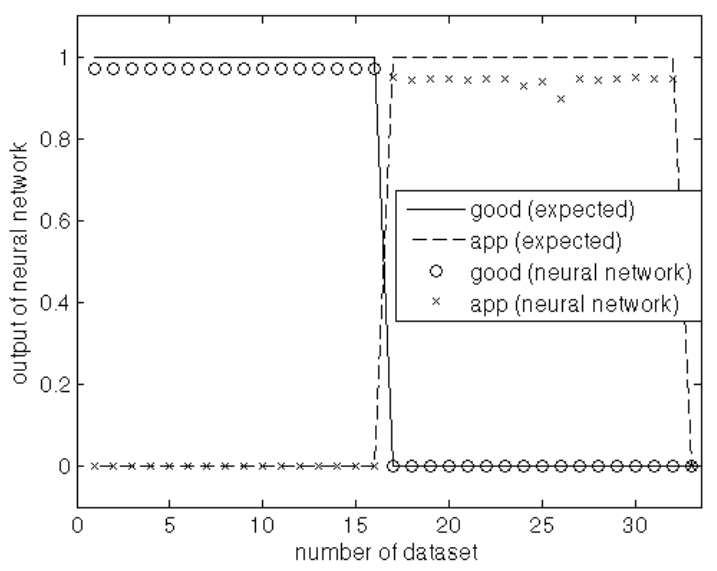

Figure 8: Classification result for an undamaged pump (good) and axial piston play (app). Lines denote the expected output of the NN for ideal classification. Circles and crosses denote classification results of measured data.

standard deviation or skewness were defined and applied to specific harmonics arranged in groups of at least 7 . If the groups are thoroughly composed, meaningful features can be extracted.

With subject to complexity a neural network (NN) was employed to classify the condition of pumps. A great advantage is the ability to train such a network with simulation data instead of experimental data, which would cause higher effort. However, in most cases measurement data of undamaged devices should be available. It is therefore reasonable to train the $\mathrm{NN}$ with those measurements and in addition with model data of all simulated defects and conditions. In our case we modeled an undamaged pump as well as one with axial piston play and presented both simulation and measurement (undamaged) data to the $\mathrm{NN}$ for training. The $\mathrm{NN}$ accepted 12 features as input and had 3 output neurons. Thus, 3 classes could be separated, but we used only two of them for an undamaged pump and axial piston play.

The classification results are presented in Figure 8. Only measurement data was presented to the $\mathrm{NN}$ for testing. A value of 1 at an output neuron means that a signal is strongly related to the specific class. Smaller values indicate a lower probability of class membership. The classification works well for our presented model and is suitable for a CMS.

\section{Conclusions}

In this paper we presented a model-based method to support the development of condition monitoring systems (CMS). The basic idea is to replace an experimental setup by simulation or at least to reduce the experimental effort during development of a CMS. For demonstration an axial piston pump with at least two signs of wear was modeled. The simulation results agree comparatively well with experimental data. Modeling has several advantages compared to experimental setups. The investigation of defects is much more flexible and one is able to find correlations between those defects and detected signals. This approach also facilitates the development of improved signal processing techniques and the generation of better features for classification.

\section{Acknowledgement}

This project was funded in part by the German Federal Ministry of Education and Research (BMBF). Associated partners are GEMAC GmbH (Chemnitz), Fraunhofer Institute IIS (EAS, Dresden) and Lenord+Bauer \& Co. GmbH (Oberhausen).

\section{References}

[1] Ming Liu, Dynamisches Verhalten hydrostatischer Axialkolbengetriebe. Bochum, Germany: PhD thesis, Institute Product and Service Engineering, Ruhr-Universität Bochum, 2001

[2] Liang Chen, Model-based fault diagnosis and fault-tolerant control for a nonlinear electrohydraulic system, PhD thesis, TU Kaiserslautern, 2010.

[3] Dymola 7.3, Dassault Systèmes

[4] Fritzson P., Principles of Object-Oriented Modeling and Simulation with Modelica 2.1, IEEE Press, 2004

[5] A. Roccatello, S. Mancò and N. Nervegna, Modelling a Variable Displacement Axial Piston Pump in a Multibody Simulation Environment, J. Dyn. Sys., Meas., Control, 129(4):456, 2007.

[6] Zoebl H., Kruschik J., Strömung durch Rohre und Ventile, Springer-Verlag Wien, Austria, 1978

[7] R. Petrovic, Mathematical Modeling and Experimental Research of Characteristic Parameters Hydrodynamic Processes of a Piston Axial Pump, Journal of Mechanical Engineering, 55(4):224-229, 2009

[8] MATLAB® 2010a, The MathWorks, Inc. 